\title{
The study on Surface organic modification of silicate glass
}

\author{
Sun Yiming ${ }^{1}$ a , Gong Depeng ${ }^{2}$ b, Ma Tengfei $^{3}$ c, Yang Ying ${ }^{4}$ d, Sun
}

\author{
Chengzhang ${ }^{5}$
}

\author{
(1,2,3,4,51.School of Materials Science and Engineering,Hubei University of Technology;Hubei \\ Wuhan430068,China;2. Key Laboratory of Green Materials for Light Industry,Hubei University of \\ Technology ,Hubei Wuhan430068,China)

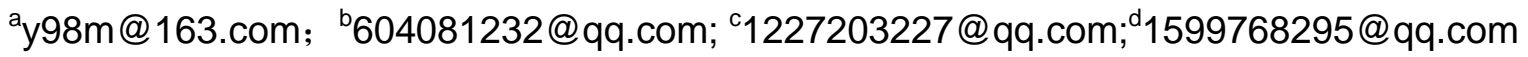 \\ e154410098@qq.com
}

Keywords: hydrophobic modification; silicate glass; acrylic acid

Abstract. The hydrophilic acrylic acid was used to modify the glass by grafting acrylic acid onto the plasma treated glass surface under the catalyzer of $\mathrm{H}_{2} \mathrm{SO}_{4}$. The results were analyzed from two aspects of composition and structure by attenuated total reflection FTIR ( ATR-FT-IR) and tensiometer ,respectively. Research shows that acrylic acid was grafted onto the glass successfully;moreover, the competition between grafting reaction of carboxyl and hydroxyl groups and self polymerization of acrylic acid had close relationship with the concentrations of sulfuric acid, when the concentrations of sulfuric acid was $14 \%$, the hydrophobic effect was the best.

\section{Introduction}

With the continuous improvement of living standards, hydrophobic surface has very important applications in many fields, such as anti-fog coatings, anti-icing coating, self cleaning surface, anti fouling surface, antibacterial surface,etal[1], and especially the hydrophobic glass has been rapid developed . Hydrophobic glass curtain wall can effectively reduce cleaning costs [2], the self -cleaning ability of the glass surface of the solar cell assembly will have a positive effect on the stability of the solar cell conversion efficiency[3].

By studying the hydrophobic lotus leaf, the combination of appropriate surface roughness and surface chemistry is responsible for the hydrophobicity and self-cleaning properties.[4]Therefor,there are two steps of obtaining hydrophobic glass surface:first step is the fabrication of appropriate roughness on the low-surface-energy materials surface,second step is chemical modification by decorating low-surface-energy materials, such as applying a layer of hydrophobic coating,grafting the low-surface-energy chemical materials e.g. fluorine compounds, silicon salt, organic polymers; .Dong Yueguo[5]used the different fluorinated acrylic ester monomer to modify the glass surface, and found 2,2,3,4,4,4-Hexafluorobutyl acrylate and 2-(Perfluorohexyl)ethyl methacrylate had better effect of hydrophobic glass surface than others,but their contact with water was not more than $120^{\circ}$.Li Qingliu et al.[6]combined the surface roughness and surface chemical modification, the $1 \mathrm{H}, 1 \mathrm{H}, 2 \mathrm{H}, 2 \mathrm{H}$-perfluorodecyltriethoxysilane was used to decorated rough surface after sanding glass surface and found that the surface contact angle reached 
127.9 ${ }^{\circ}$. Cao Geng et al.[7] use hydrofluoric acid (HF) solution to pre processing of glass surface, forming micro nano structure, then formed hydrophobic molecular film on the surface of the glass by eighteen alkyl three (OTS) molecules self-assembled , and prepared transparent hydrophobic glass with a contact angle of $107^{\circ}$. Chen Guoping [8] coated transparent hydrophobic fluorine alkyl silane (FAS) - SiO2 membrane on the glass surface by sol-gel method,the results showed that with the increase of the number of $\mathrm{SiO} 2$ film, the hydrophobicity of the glass was more better; the contact angle was $122^{\circ}$ when the glass surface was coated with $\mathrm{SiO} 2$ and FAS.

In summary, the chemical modification of fluorinated compounds and other low surface energy substances can get hydrophobic effect, and the surface contact angle can reach $120^{\circ}$ or so ,but containing fluorine material is expensive and is unfriendly to environment because of slow degradation; the fabrication of rough surface by low surface energy is very difficult in practical applications as well,not only because of high cost of material,harsh preparation conditions, but also due to low strength, poor durability,poor uniformity and bad weather resistance of hydrophobic surface . The template method is the most easy to control the surface uniformity in the preparation method of the existing hydrophobic thin film,however,most of the template is made by the soft template of the lotus leaf which has a lot of problems to be solved,e.g the durability is poor,it is not easy to repeat the use, the area can not meet the requirements of mass production.

In this paper ,the hydrophilic acrylic acid was used to modify the silicate glass surface, and obtained hydrophobic effect,which was almost no reports at home and abroad.Because of the advantages of low cost, simple process, high production efficiency, this method can realize industrial production, and has important application prospects; furthermore because of the dual function of acrylic acid, after grafting the acrylic acid onto the glass surface,by controlling the reaction conditions, another functional group can be used to carry out the next step of the reaction.

\section{Experiment}

\section{Materials and Apparatus}

Materials: Acrylic acid: CP, Tianjin Kermel Chemical Reagent Co. Ltd.; sulfuric acid: CP, Xinyang Chemical Reagent Factory;30\% hydrogen peroxide: AR, National Pharmaceutical Group Chemical Reagent Co., Ltd;Anhydrous alcohol: AR, National Pharmaceutical Group Chemical Reagent Co., Ltd;sodium hydroxide,AR,Xilong Chemical Limited by Share Ltd;hydrochloric acid: $\mathrm{CP}$, Xinyang chemical reagent factory; distilled water,homemade.

Apparatus:attenuated total reflection FTIR ( ATR-FT-IR) was used to analyze the composition of the glass surface; tensiometer from Germany,KRUSS was used to measure contact angle.

\section{Experiment method}

Pretreatment of glass surface

The glass was cleaned by ordinary detergent and then flushed by large quantities of distilled water, second the cleaned glass was put into the beaker full of anhydrous ethanol to ultrasonic treat for $1 \mathrm{~h}$,and then flushed three times by distilled water,third, the anhydrous ethanol changed into distilled water,did as the second step, and then blew dry standby.

Enrich hydroxyl groups on the surface of glass

In order to make the graft of acrylic acid more efficient,it is very necessary to enrich hydroxyl on the surface of the glass first.Usually,three method of riching hydroxyl was applied:acid treatment, alkali treatment, plasma treatment. 
Glass surface grafting acrylic acid

In this reaction,the glass film with enriching hydroxyl, 30ml acrylic acid solution and the amount of concentrated sulfuric acid were put into the test tube, then stirred one hour at 70 degrees.By making use of the carboxyl groups and hydroxyl groups on the surface of the glass, it can realize the grafting acrylic acid on the glass surface ; by using the carbon -carbon double bond , further reaction can be used to improve the hydrophobic property.Figure 1 is the basic principle of the reaction equation.

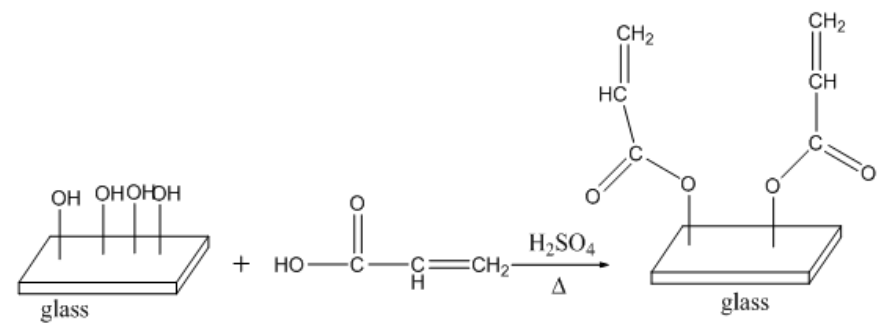

Fig.1.The grafting reaction

\section{Results and discussion}

\section{The result and discussion of ATR FTIR}

ATR-FTIR obtained the organic components of sample surface structure information by the reflected signals from the sample surface, which has the characteristics of high detection sensitivity, small measurement area.Figure two is the ATR-FTIR spectrum, $a$ is a rich hydroxyl of the glass, $b$ is blank clean glass,c is grafted acrylic glass.

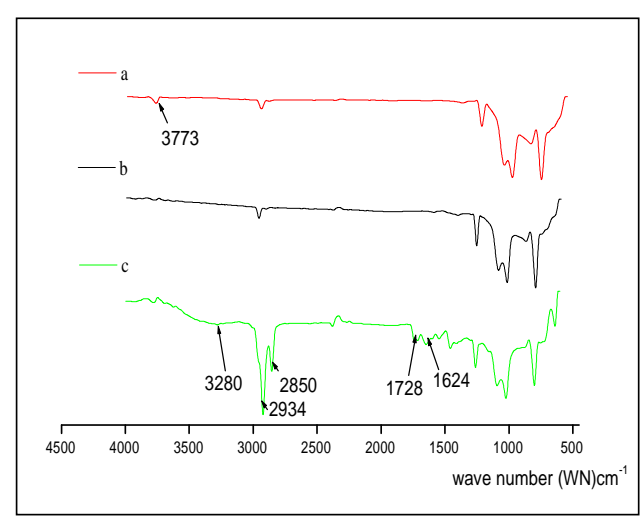

Figure. 2. the ATR-FTIR spectrum.

(a) enrich hydroxyl of the glass; (b) blank clean glass;(c) grafted acrylic glass.

By comparing the $a$ and $b$, we can find a new peak in $3773 \mathrm{~cm}^{-1}$, which was considered the absorption peak of the double hydroxyl group on the silicate surface[9]. It can be clearly seen that after the enrichment of hydroxyl, the surface of silicate glass is indeed a hydroxyl group, and it is mainly the double hydroxyl groups. When comparing the a and c,we also found obviously difference: $1624 \mathrm{~cm}^{-1}, 1728 \mathrm{~cm}^{-1}, 3280 \mathrm{~cm}^{-1}$ absorption peak is the bending vibration of double bond ,stretching vibration carbon oxygen double bond,stretching vibration of $\mathrm{C}-\mathrm{H}$ bond on carboncarbon double bond, respectively,which is the characteristic absorption peak of acrylic acid.it showed that the acrylic acid had been grafted onto the glass successfully.In addition ,2934 $\mathrm{cm}^{-1}$ absorption peak is stretching vibration of saturated $\mathrm{C}-\mathrm{H}$, We proposed that this is due to the addition of carbon-carbon double bond between the acrylic acid molecules which grafted the double hydroxyl group on the silicate surface.It is the condensation reaction between the carboxyl group of acrylic acid and the hydroxyl group of the glass, so that the hydrophilic groups disappear, so the 
surface of the glass is hydrophobic.

\section{The result and discussion of contact angle}

Contact angle is not only the determination of wetting quality, but also the determination of the surface hydrophobic property.Figure 3shows the change of surface contact angle under different treatment methods.a is the result of enriching hydroxyl group, $b$ is the result of the grafting of acrylic acid after the enriching hydroxyl group, broken line express the difference between the contact angle of the two processing before and after.It is obviously found that the contact angle by plasma treating decreased most ,however,the contact angle by acid treating of the grafting of acrylic acid after the enriching hydroxyl group increased most.We analyze that the surface of the glass after the plasma treatment is not all hydroxyl group,may have a variety of oxygen-containing groups[10]which shows hydrophilic property, but can not participate in the graft reaction.In addition,acid treatment not only enriched hydroxyl group ,but also increased the roughness of the glass surface by corrosion. Therefor, though the contact angle of plasma treatment was smaller than that of acid treatment, the contact angle of the grafting of acrylic acid was contrary.Besides,it's evidently found that the contact angle of the grafting of after the enriching hydroxyl group was generally improved,especially the plasma treatment and acid treatment were increased by $73.24 \%$ and $114.86 \%$,respectively.

Sulfuric acid as the catalyst in the reaction can not only promote the grafting reaction, but also can promote the self polymerization of acrylic acid molecules .In order to find the optimal reaction conditions of grafting acrylic acid onto the glass,experiment was conducted under different concentrations of sulfuric acid,then contact angle was measured.Figure 4 shows the variation of the contact angle at different concentrations of sulfuric acid.a is not processed , b is treated with plasma. It's obviously found that the contact angle was bigger after plasma treating. The tendency that with the increase of concentration of sulfuric acid, the contact angle increases at first and then decreases can be seen from the diagram as well.Through the analysis of the results, we consider

that at the low concentration of sulfuric acid,the sulfuric acid can not play a catalytic role; with the adding of sulfuric acid concentration, sulfuric acid can play a role in dehydration in the reaction of carboxyl and hydroxyl groups, promoting the reaction;when the sulfuric acid concentration was further increased, the sulfuric acid not only played the role of dehydration, but also was beneficial to the self polymerization of the acrylic acid molecules, thereby forming a more hydrophobic structure;however, when the concentration of sulfuric acid was further increased, the acrylic acid is more favorable for self polymerization, and inhibited.the grafting reaction. 


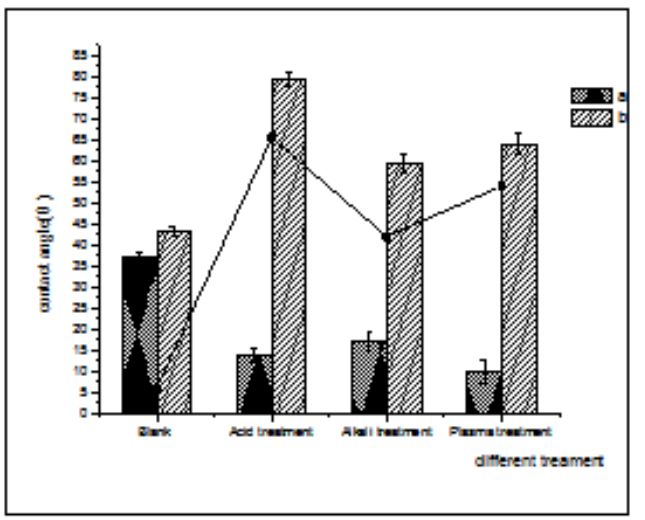

Fig.3.The change of surface contact angle under different treatment methods.(a)enriching hydroxyl group;(b)the grafting of acrylic acid;(broken line) difference of the contact angle of the two processing

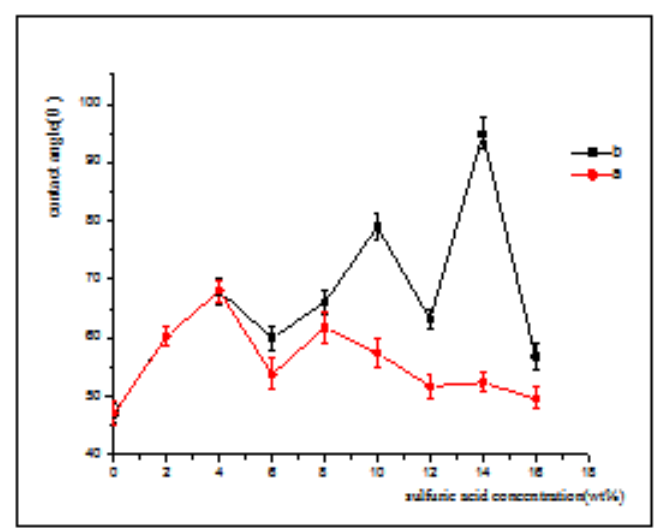

Fig.4.the variation of the contact angle at different concentrations of sulfuric acid. (a) not processed ;(b) treated with plasma

\section{Conclusion}

The surface organic modification of silicate glass was studied and analyzed from two aspects of composition and structure.The result of ATR-FTIR can prove acrylic acid had been grafted onto the glass successfully.By analyzing the contact angle result,it can prove that three method of enriching hydroxyl was effective,and the plasma treatment worked best;sulfuric acid as the catalyst in the reaction can not only promote the grafting reaction, but also can promote the self polymerization of acrylic acid molecules, when the concentrations of sulfuric acid was $14 \%$, the hydrophobic effect was the best.

\section{Reference}

[1]Chen Jun, Wang Zhenhui, Wang Wei: Materials China. 07(2013), p. 399-405

[2] Sato.O, Kubo.S and Gu Z.Z: Account of Chemical Research . 42(2009),p. 1-10

[3] Asmatulu R, Ceylan M and Nuraje N: Langmuir . 27(2011), p. 504-507

[4]]Akira Nakajima, Kazuhito Hashimoto and Toshiya Watanabe: Monatshefte Fur Chemie. 132(2001), p. 31-41

[5]Dong Yueguo: Modification Of Glass Surface By Silanecoupling Agent. Beijing University of Chemical Technology(2014).

[6]Li Qingliu, Liu Ming, Ma Dong etal: China Surface Engineering. 03(2014), p.95-100.

[7]Cao Geng, Pan Meiying, Zhong Rui, etal: Journal of Sichuan University (Natural Science Edition). 04(2014),p. 804-808

[8]Chen Guoping: Silicone Material. 03(2007), p. 125-128

[9]Hu Fuzeng: Materials Surface and Interface. East China University of Science and Technology Press.(2008), p. 128-130

[10]Tian Jiwen: Material Review.03 (2005), p. 10-12 